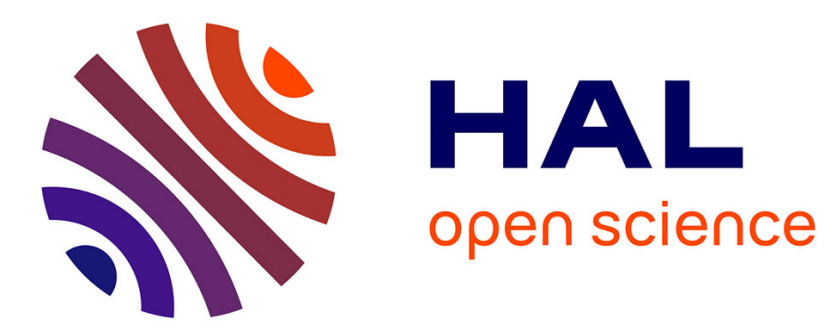

\title{
Le psychodrame du football français
}

\author{
Patrick Mignon
}

\section{To cite this version:}

Patrick Mignon. Le psychodrame du football français. Revue Esprit, 2010, 367, pp.6-22. hal02058915

\section{HAL Id: hal-02058915 https://hal-insep.archives-ouvertes.fr/hal-02058915}

Submitted on 6 Mar 2019

HAL is a multi-disciplinary open access archive for the deposit and dissemination of scientific research documents, whether they are published or not. The documents may come from teaching and research institutions in France or abroad, or from public or private research centers.
L'archive ouverte pluridisciplinaire HAL, est destinée au dépôt et à la diffusion de documents scientifiques de niveau recherche, publiés ou non, émanant des établissements d'enseignement et de recherche français ou étrangers, des laboratoires publics ou privés. 


\title{
Le psychodrame du football français
}

\author{
Patrick Mignon *
}

Article paru dans : Esprit, août-septembre 2010.

Même s’il s’agit de football, le sang-froid est de rigueur en parlant de la Coupe du monde. Quand la France gagne, il faut échapper à l’illusion lyrique qui fait de l'équipe le miroir de la société, comme en 1998, quand l'intégration semblait célébrée par la réussite sur le terrain. Quand elle perd, comme cette année, par absence de jeu et ambiance de télé-réalité, il faut résister à d'autres surinterprétations : « la France black blanc beur a disparu, les racailles ont pris le pouvoir », ou à l'ambiance de chasse aux sorcières : au choix, Escalettes, Domenech, Anelka, etc. Tout a été dit, toutes les hypothèses ont été lancées. Essayons simplement ici de mettre en évidence des mécanismes qui, pour échapper au piège de la surinterprétation, permettent de comprendre comment les choses se passent dans un grand sport professionnel comme le football pour savoir ce qu'on peut en attendre, et du sport en général, pour comprendre la société et, éventuellement, agir sur elle.

\section{Médiatisation et sensibilité du corps social}

Durant la dernière décennie, les événements sportifs sont devenus non seulement les programmes télévisés les plus populaires mais aussi la principale source de sujets pour les journaux télévisés, devant l'information économique, politique ou culturelle. Entre 2000 et 2009, le sport a représenté 7,7\% de l'offre des journaux télévisés avec 24304 sujets, contre 21936 pour l'information politique. Le football concentre à lui seul plus de $35 \%$ des sujets consacrés au sport avec 7946 sujets sur les dix années précédentes. Le tennis, deuxième sport le plus médiatique, enregistre $10 \%$ de part de marché ${ }^{\text {. }}$

Cette situation est révélatrice de la place occupée par le sport dans les imaginaires sociaux et des questions qu'il permet de traiter concernant la part attribuable au mérite ou à la chance et aux relations à établir entre initiative individuelle et organisation collective dans la réussite d'une équipe. Le football et la Coupe du monde sont, pour ces raisons, des forces fédératrices dont, faute de détailler les formes et la diversité des contenus, on peut au moins mesurer l'impact, même s’il n’est pas en France aussi important qu'en Angleterre, en Argentine ou au Brésil.

Cette hypermédiatisation a pour effet de donner le sentiment qu’à travers une mésaventure sportive, c’est l'ensemble du corps social qui est représenté, appelant des réactions immédiates, interprétations et accusations, censées répondre aux interrogations et aux émotions des spectateurs et surtout des téléspectateurs.

Elle repose sur la multiplication et la diversification des interprétations liées à la concurrence entre les différents médias et fait de la scène sportive un lieu fondamental d'investissement pour la mise en scène du politique. Celui-ci en a fait un moyen de communication, de mise en scène de soi et de manifestation d'un style de gouvernement. Cette situation est renforcée par le fait que le Président actuel se présente comme un vrai amateur de sport et se préoccupe personnellement du fonctionnement de l'équipe de France comme en témoigne son rendez-vous avec Thierry Henry au détriment de réunions de préparation du G20 avec des organisations non gouvernementales.

\section{Les particularités de la Coupe du monde}


Qui s’intéresse au football en France ? Environ un tiers de la population âgée de plus de 15 ans (et même plus, si l'on compte les enfants dès 8-10 ans). Un autre tiers de la population s'intéresse à la Coupe du monde, ce qui signifie que le destin de l'équipe de France est plus mobilisateur que celui de l'OM ou de l'OL. La Coupe du monde, c'est-à-dire la compétition entre des équipes représentant des nations, ou plutôt des fédérations nationales, est l'occasion de se mobiliser autour des représentants de la nation. Il y a donc un intérêt patriotique qu'on se gardera d'analyser comme un chauvinisme de fermeture : la finale a attiré 14,1 millions de spectateurs, troisième score derrière ceux de l'équipe de France contre le Mexique et l’Uruguay (15 millions à chaque fois).

Pourquoi s'intéresse-t-on encore à la Coupe du monde de football ? Pour les couleurs donc, et, bien sûr, par amour du football qui fait qu'on veut savoir quelle équipe est la meilleure, quel style de jeu l'emportera. Pourtant, dans le contexte du football contemporain, elle a ses détracteurs. Ceux-ci se demandent si le niveau technique est vraiment aussi élevé que dans le football des clubs, des grands championnats d'Angleterre ou d'Espagne ou de la Champions' League ? Ils n’ont pas tort car tous les grands joueurs ne sont pas présents lors de la compétition mondiale pour peu que leur sélection nationale ne se soit pas qualifiée et, surtout au début, parce que les équipes nationales doivent construire sur la durée de la compétition, quatre semaines et un peu de préparation, un jeu et un collectif que les clubs produisent sur une saison voire plusieurs et, pour certaines, en faisant venir chez elles les meilleurs joueurs du monde. Mais ce n'est pas grave et c’est justement l'intérêt du Mondial. Le chauvinisme s'efface si l'équipe nationale est éliminée : la question du jeu et de la cohésion du collectif, et donc du style, devient alors centrale. Le chauvinisme survit néanmoins sous sa forme comparative : pourquoi les autres font-ils mieux que nous ? Cette comparaison a peut-être alimenté le sentiment nourri par sept Français sur dix de vivre dans un pays " en déclin ${ }^{2}$ ». Car on y mesure l'état d'esprit du collectif rassemblé et le savoir-faire jouer ensemble des différents entraîneurs. La Coupe du monde, comme la Coupe d'Europe, met à l'épreuve cette question comparative : comment chacun s'y prend-il pour réussir dans un grand événement?

\section{Qu'est-ce qu'une compétition?}

Une compétition qui s'étire sur un mois se trouve à la rencontre de diverses séries de chances et de décisions. Le fait sportif est par définition aléatoire. Le football est un sport, c’est-à-dire un jeu. Dire cela, ce n'est pas seulement rappeler une morale élémentaire qui invite à ne pas y engager tous ses biens ni à y sacrifier toutes ses valeurs, mais aussi que les résultats d'une équipe sont autant l'effet de la succession des chances que des décisions, bonnes et mauvaises, prises au cours du match. Les économistes qui se sont fait une spécialité de la prévision des résultats sportifs plaident pour le succès s’ils approchent les $50 \%$ de bons pronostics. L'opposition de deux collectifs arbitrée par un homme multiplie les chances et les occasions d'erreur ou de réussite. L'attribution d'un succès ne peut se faire seulement en distinguant les mérites des individus mais aussi en acceptant la part de la chance et des routines dans le résultat final.

Que se passe-t-il si, au lieu d'être mené au score, on a ouvert la marque ? Quelle influence ce carton jaune immérité a-t-il sur le déroulement de la rencontre ou de celle qui va suivre ? On peut gagner grâce aux tirs au but parce que le goal a glissé ou avoir un parcours facile dans une compétition grâce au tirage au sort. Autre hasard : l'inégale répartition des talents dans les générations. À certaines époques, un pays peut avoir plusieurs numéros 10 de grand talent et à d'autres aucuns, ou avoir à différents postes ce qui se fait de mieux dans le domaine du football. Mais cette inégale répartition des joueurs talentueux vaut aussi pour les sélectionneurs : peut-être 
a-t-il mal cherché, mal choisi, peut-être était-il entouré de mauvais conseillers, qu’il avait luimême choisis ou qu'on lui avait imposés ? Peut-être y a-t-il, comme pour les joueurs, des générations dorées d'entraîneurs ? Il ne manque pas de bons esprits pour considérer que c'est l'entraîneur qui fait la différence ou que ce sont, à l'inverse, les joueurs qui organisent l'équipe. C'est une explication qu'on donne volontiers des deux victoires de 1998 et 2000 ou du bon parcours de 2006. Pour toutes ces raisons, les résultats relèvent pour une bonne part du hasard, plus que d'une organisation rationnelle. Cette incertitude fait l'intérêt du sport et du football, en particulier où ce n'est pas toujours le meilleur, intrinsèquement ou dans sa capacité à tirer parti des chances, qui gagne.

\section{L’héritage de 1998}

Pour comprendre le psychodrame national et évoquer des dysfonctionnements qui se sont multipliés au sein de l'équipe de France, il faut remonter à 1998. La victoire de 1998 a été une divine surprise. Pour le monde du football, ce fut une surprise parce que les sceptiques étaient nombreux, parce que les résultats d'avant la compétition n’étaient pas convaincants, parce que beaucoup partageaient la méfiance vis-à-vis du style mis en place par Aimé Jacquet. Mais l'équipe a gagné et a comblé d'aise ceux qui y croyaient dur comme fer et créé un lien entre tous les nouveaux adeptes du football : on peut, lors d'une coupe du monde, se mettre derrière l'équipe de France, pour différentes raisons, parce qu'elle peut gagner, qu'elle est sympathique, qu'elle joue bien et surtout qu'elle permet d'entrer dans le jeu mondial des supporters. La victoire dans le championnat d'Europe 2000 paraissait avoir confirmé ces attentes.

Pour la classe politique aussi, ce fut une chance. À travers le mythe de la France black, blanc, beur, les Bleus de 1998 ont représenté l'image d’une société où les inégalités et les discriminations pouvaient être surmontées par les valeurs du sport. La victoire et l'organisation de la compétition ont donné l'idée que le sport était susceptible de dynamiser la croissance, de transformer le visage des villes et de valoriser l'image de la France à travers le monde. Avant la coupe, la presse étrangère se gaussait d'un pays rongé par les grèves, bureaucratisé, sans culture du football. Qu'a-t-on admiré après ? La formation à la française et les effets de la méritocratie sportive, les grèves qui s’arrêtent juste au début de la compétition, le TGV qui transporte journalistes et supporters, l'accueil dans les villes et la sécurité des matches, le rôle de l'État à travers ses cadres techniques du football...

Cette victoire a laissé un héritage à gérer. Un héritage sportif, c'est-à-dire la gestion des conditions d'une présence régulière à haut niveau d'une équipe de France de football. Un héritage social, c'est à dire la prise en compte et la compréhension du rôle du sport dans les processus d'intégration sociale, à travers l'accompagnement du rôle social, culturel du football qui suppose l'accroissement du nombre de licenciés, l'amélioration de la vie sociale dans les clubs, le maintien d'un haut niveau technique et éducatif dans ces clubs, l'accueil des nouveaux adeptes (notamment les jeunes filles), et aux nouvelles attentes, moins compétitives que de sociabilité, la considération donnée aux supporters de l'équipe de France. C'est un héritage politique, c'est-àdire qui prend au sérieux les enjeux du football en luttant contre le clientélisme et l'instrumentalisation. C'est un héritage économique, consistant à contrôler et à développer les ressources nées des succès de l'équipe nationale et d'apprécier sa juste contribution à la vie économique.

Ces héritages n’ont pas été gérés de manière égale ou conséquente : la gestion de la rente représentée par l'équipe de France a donné lieu à une mobilisation plus conséquente que la transformation des conditions d'accueil dans les clubs ou l'appréciation de la nature du lien entre 
l'équipe et ses supporters. Ces trois dernières années, le nombre des licenciés a baissé. Le football reste encore la fédération la plus puissante, mais le désamour des pratiquants sportifs visà-vis des clubs est fait du manque d'attractivité du plus haut niveau et du manque d'attractivité des structures de base et du découragement qu'elles peuvent provoquer parmi les nouveaux adeptes ou parmi les plus fidèles qui ne se reconnaissent plus dans les évolutions de la pratique.

\section{L’équipe de France comme produit}

Un club professionnel de football est une PME qui paie des salaires extravagants à quelques-uns de ses salariés. Ces salaires payés aux stars sont possibles grâce à la médiatisation du sport qui constitue un des principaux produits d'appel des grandes chaînes de télévision. Les grands événements, type Coupe du monde de football ou jeux Olympiques, réalisent parmi les plus fortes audiences télévisuelles. Les droits de retransmission des différents championnats qui sont reversés aux clubs constituent, en France, plus de la moitié et, pour certains, la quasi-totalité de leurs ressources. On a ainsi pu parler de télé-dépendance pour définir l’économie des clubs de football.

C’est aussi le cas des sélections nationales. Ainsi, avant la Coupe du monde, l'audience télévisée la plus forte enregistrée en 2009 est détenue par le match France-Irlande qui a réuni plus de 11,7 millions de téléspectateurs. Pour la Fédération internationale de football association (Fifa), la cession des droits de retransmission est particulièrement lucrative, puisque pour la Coupe 2010 devrait lui rapporter plus de 2 milliards d'euros. En France, TF1 a déboursé 120 millions d'euros pour obtenir les droits de diffusion qui seront revendus aux autres chaînes, TF1 gardant les matches de la France considérés comme les plus porteurs. La médiatisation a donc pour effet de drainer les sponsors qui vont s'engager derrière l'événement, à travers l'achat d'écrans publicitaires, et du sponsoring des fédérations, des sportifs ou des équipes qui les représentent. Ce sont ces contrats qui permettent le versement de primes de participation et de récompenses aux joueurs.

L'équipe de France est ainsi devenue un produit, elle est la principale ressource de la Fédération française de football (FFF), environ $40 \%$ de ses recettes grâce aux droits versés par les télévisions et par les sponsors. L’équipe de France, comme toutes les équipes de football, a donc pris le tournant de la médiatisation. Comme les clubs, l'équipe nationale doit être présente dans les grands événements pour attirer, garder, changer de sponsors (Nike a supplanté Adidas comme fournisseur d'équipement, en offrant près de 43 millions d'euros, sur un budget d'environ 200millions d'euros, par an).

Lorsqu'on dit que les grandes fédérations ne sont pas adaptées " pour manier des intérêts économiques aussi puissants ", ce n’est pas sûr dans ce cas précis. Mais on peut se demander si la défense du produit ou de la marque équipe de France n'a pas pris le pas sur l'ensemble des autres enjeux. En effet, le jeu n'a pas suivi, la qualité du jeu ne permettait pas de gagner ni de mobiliser les supporters.

\section{Pourquoi peut-il y avoir des dysfonctionnements dans une fédération?}

Si tout est affaire de chance et de cycles, parler des fautes des individus ou des dysfonctionnements n'aurait donc pas de sens ? Certains ont pourtant l'air, mieux que d'autres, de surmonter ces aléas. Par exemple, en assurant une présence régulière dans les compétitions, comme c'est le cas de l'Allemagne qui détient un record avec onze participations en phase finale de Coupe du monde et qui a donc figuré honorablement avec des équipes brillantes aussi bien 
qu'avec des équipes moyennes sans joueur vraiment talentueux. Il y a donc de la chance, des cycles, mais aussi des décisions et des politiques pour limiter les effets du hasard.

La question est donc : en quoi la politique de l'équipe de France n’a-t-elle pas pu assurer la continuité sportive qui comprend la présence régulière à haut niveau et la fidélisation des supporters par un jeu agréable, selon les goûts, parce que l'équipe gagne avec brio, joue sa chance, tombe avec les honneurs, fait plaisir, mouille le maillot, rend fier, fait honneur, gagne malgré tout, etc. ?

La performance en sport d'élite est une production collective, c'est un système d'action dans un univers de compétition. On peut distinguer deux niveaux qui concourent à cette production : le niveau technique qui est directement impliqué dans la production de la performance qui comprend l'entraîneur-sélectionneur, et son équipe, nommée par la fédération ; les joueurs sélectionnés ou sélectionnables ; tous les cadres techniques de la fédération regroupés au sein de la Direction technique nationale (DTN) qui sont les garants de la formation des joueurs par la qualité de la formation des entraîneurs et des éducateurs qu'elle a en charge, en plus de l'encadrement des équipes nationales, à l'exclusion de l'équipe A; mais on doit aussi introduire tous les candidats potentiels au poste de sélectionneur qui sont, soit membres de la DTN, soit entraîneurs travaillant sur le marché national et international des clubs professionnels.

Le deuxième niveau est le niveau politique qui prend des décisions, par exemple de choisir telle ou telle personne pour devenir le sélectionneur de l'équipe de France. C'est la part qui revient aux élus de la fédération, le président, élu par les représentants des structures locales (ligues et districts), nationales et professionnelles du monde du football, et le conseil fédéral qui représente l'ensemble des familles du football (amateurs, professionnels, éducateurs, arbitres, joueurs) qui détient les pouvoirs de direction et nomme les cadres les plus importants, les directeurs par exemple. S'il y a dysfonctionnement, c'est parce qu'il existe des tensions provoquées par les enjeux de pouvoir autour de l'équipe de France et de sa maîtrise : on peut imaginer sans mal que la vision portée par les représentants du monde du football professionnel, self image de compétence et contrôle des ressources du produit " équipe de France ", n’est pas la même que celle du monde amateur, émanation du monde ordinaire du football, partage de l'aura de l'équipe et contrôle des ressources (c'est-à-dire comment se partager les retombées économiques de l'équipe de France).

Il existe aussi des tensions entre le politique et le technique, chacun considérant qu'il détient la légitimité pour définir la politique fédérale. Sachant que, dans toutes les fédérations, l'équipe nationale est l'élément phare, celui qui apporte réputation et ressources, on comprend qu'il puisse y avoir des tensions. Dans le football, le sélectionneur est embauché par le président, même si, comme souvent, cas d'Aimé Jacquet, Gérard Houllier ou Raymond Domenech, le sélectionneur vient de la DTN. On peut donc, dans l'affaire, reprocher à la DTN le manque de bagage technique des joueurs, leur manque de sens du collectif, leur absence d'inventivité ou de passion puisqu'elle a formé dans son centre de Clairefontaine une partie des joueurs de l'équipe de France (dont Nicolas Anelka), et les éducateurs et entraîneurs qui officient dans les centres de formation des clubs professionnels. C'est d'ailleurs ce que reconnaît l'actuel responsable de la DTN, Gérard Houllier ${ }^{3}$, ou d'autres responsables de cette structure comme Yannick Stopyra, ancien coéquipier de Platini en équipe de France. Mais on pourra aussi se demander pourquoi les analyses proposées après coup de la mésaventure de l'équipe de France ne l'ont pas été avant : à savoir que le sélectionneur était en train de travailler tout seul et que « l'équipe de France était devenue paranoïaque ».

\section{La place de l’État}


A-t-on donc eu raison de taper fort sur la fédération, son président, les faiblesses du bénévolat, etc., à travers les propos des ministres en charge du sport et du président de la République ${ }^{4}$ ? Les remontrances de la Fifa au gouvernement français concernant son interventionnisme dans les affaires du football, en rappelant notamment qu'une sélection nationale représente une fédération et non un État, ont politisé l'affaire et invite à poser la question de la place de l'État dans le sport professionnel. Ici encore, il faut distinguer différents niveaux. D’abord, celui de la délégation par l'État d'une mission de service public aux fédérations sportives qui comprend, outre le développement du sport, la présence de la France au niveau international. La place de l'État, ici, se traduit par la nomination, au sein de la fédération, d’agents chargés de mettre en oeuvre la politique sportive et qu'on retrouve donc dans la DTN ou dans d'autres services de la fédération avec comme mission de faire avancer des dossiers importants pour le ministère tels que la lutte contre la violence dans le football amateur ou la participation du football dans les différents dispositifs d'insertion par le sport.

Le ministère des Sports n'a pas su s'adapter à la professionnalisation du sport et l'affaire remonte à loin. Le ministère des Sports n’a pris que récemment et, à la marge, le tournant du sport professionnel, plus sur son versant politique qu'administratif par la croyance dans l'exemplarité du sport professionnel comme modèle d'organisation sociale et politique, sous l'effet de l'arrivée d'un Président « sportif » qui voit dans le sport un modèle de comportement et qui a favorisé, après quelques autres, l'ouverture au sport professionnel. L’intervention récente repose plus sur la fascination pour la réussite économique de quelques grands patrons, comme Jean-Michel Aulas ou Arnaud Lagardère vus comme modèles d'excellence de l'esprit d'entreprise, et leurs investissements, plus ou moins heureux, dans le sport et comme la résultante d'un lobbying réussi de la part des secteurs des sports professionnels, où le football se trouve au premier rang à travers la mise en avant de la « réussite » du business model de la Premier League anglaise. Une mise à niveau du sport français reste à faire et, si elle est faite, elle permettra de faire du sport un lieu d'investissement économique et de grandeur nationale ${ }^{5}$ par l'organisation des grands événements sportifs.

Dans son versant administratif, le ministère a longtemps nié l'existence du professionnalisme. Comme à la FFF, la culture de l'administration et des différents ministres a longtemps été une culture de l'amateurisme. Cette culture est valable tant que les contraintes spécifiques au sport d'élite telles que la médiatisation du sport, les investissements des sponsors, de l'État et des collectivités locales, les nouvelles stratégies des fédérations internationales et du CIO ne transforment pas tous les sportifs en professionnels simplement distingués entre eux par leur distribution sur une échelle de rémunération marquée par les inégalités spectaculaires qui séparent la star du football opérant en Angleterre et le médaillé d’or en lutte. Récuser la philosophie du sport professionnel a conduit pratiquement à en ignorer l'existence et empêcher d’en comprendre les mécanismes.

\section{Que signifie professionnaliser?}

Les limites du bénévolat pour gérer des questions qui supposent des compétences pointues, les effets pervers de la morale du dévouement qui fait passer pour injustes les mises en question : tout milite pour la professionnalisation des fédérations. Ce nécessaire processus continu de professionnalisation, déjà entamé notamment dans son mode de gestion de la marque " équipe de France ", ne saurait se confondre avec le passage de l'équipe nationale sous la tutelle du monde du football professionnel. À la suite de l'échec de la Coupe du monde 2010, plusieurs dirigeants 
de clubs professionnels ont demandé que la Ligue de football professionnelle (LFP) soit responsable, à la place de la FFF, de l'équipe de France. Mais l'équipe de France n'est pas et ne sera jamais un club professionnel. Les enjeux d'une équipe nationale dépassent largement ceux d'un club professionnel. Jouer en équipe nationale a une valeur symbolique qui n'existe pas en club professionnel.

Le football professionnel s'intéresse à l'équipe de France en raison de l'importante source de revenus qu'elle représente. Ainsi, sur un budget de 200 millions d'euros pour la FFF, 40\% (80 millions d'euros) ont été générés par l'équipe de France pour 39millions d'euros de charges. Le résultat brut de l'équipe de France dépasse ainsi les 40 millions d'euros. Le résultat net, après versement des primes aux joueurs, se monte à plus de 30 millions d'euros ${ }^{6}$. Les demandes de quelques présidents apparaissent ainsi comme une stratégie de captation de rente. Le transfert de l'équipe de France à la LFP lui permettrait de restaurer son équilibre financier, déficitaire au cours de la saison 2008-2009, pour un montant proche de celui du résultat net de l'équipe de France (-33millions d'euros ${ }^{7}$ ).

Mais les pays où les clubs professionnels sont plus importants que l'équipe nationale n'ont pas de meilleurs résultats, comme l'illustre le cas de l'Angleterre. Des conflits d'intérêts sur la sélection privilégiée de certains joueurs ou la non-mise à disposition des joueurs sont prévisibles et il n'est pas sûr que le monde professionnel français soit plus compétent pour diriger les joueurs opérant à l'étranger, à moins que les demandes de changement dans la fiscalité des footballeurs professionnels ne permettent de les garder sur le territoire hexagonal. Mais ce peut être aussi, cas de l'Angleterre, pour faire venir des talents d'autres pays. Il n'y a pas si longtemps, certains présidents de clubs demandaient la fin de l'obligation d'entretenir un centre de formation trop coûteux quand il est plus économique d'aller chercher des talents dans les pays où les exigences salariales sont moins fortes. Dans la perspective de l'organisation de l'Euro 2016 et du programme de construction de nouveaux stades, une confusion peut aussi s'installer en ce qui concerne l'appropriation des installations sportives. Les clubs de football professionnel réclament de plus en plus souvent la propriété ou l'usage exclusif de leur stade pour générer des recettes supplémentaires en spectateurs, marchandising et organisation d'autres spectacles que sportifs. On se dirigerait vers un modèle à l'américaine où la franchise privée fait pression sur le public, les municipalités, pour obtenir les infrastructures dont il a besoin.

Mais le monde professionnel a aussi sa part de responsabilité dans l'échec de l'équipe de France. L'argument principal pour transférer l'équipe de France à la LFP est la compétence des dirigeants des clubs professionnels. Or cette supposée compétence ne suffit pas à légitimer la direction d'une équipe ou d'une fédération. Les élus de la fédération tirent leur légitimité de leur élection, ils reçoivent en quelque sorte un mandat politique pour diriger la fédération et s'entourent pour cela d'experts qui mettent en oeuvre les politiques définies par les membres élus et qui jouent un rôle d'aide à la décision. La réalité de la compétence des dirigeants des clubs professionnels ne va d'ailleurs pas de soi. La situation des comptes des clubs professionnels affiche un résultat net négatif et le modèle financier du football professionnel comprend d'importantes faiblesses comme l'illustre la situation critique de certains clubs anglais, dont le développement était fondé sur une logique de bulle spéculative.

Par ailleurs, les joueurs, dont on critique aujourd'hui le comportement, ont été pris en charge dans des centres de formation des clubs professionnels et ont appris dès leur plus jeune âge à " trahir » leurs couleurs en raison de la concurrence entre les clubs pour s'approprier les talents prometteurs. Si l'équipe de France connaît aujourd'hui une situation aussi délicate, c'est aussi en raison de la mainmise trop importante du football professionnel sur les joueurs. À un moment où l'Europe du football, par l'entremise de l'UEFA (Union of European Football Associations), est à 
la recherche de régulations financières susceptibles d'assainir les finances des clubs, de prôner la modération et, surtout, de promouvoir une plus grande égalité des compétiteurs, il serait dommageable de casser un système qui, d'une certaine mesure, protégeait l'équipe de France. Ce serait aussi un coup dur porté au foot amateur et cela signerait surtout la fin de la solidarité entre mondes amateur et professionnel : le football professionnel existe aussi par la masse des pratiquants.

\section{Conspiration du silence}

Le choix d'un sélectionneur est une décision délicate qui ne peut échapper à l'erreur. Dans le contexte présent, il a pu être compliqué du fait de l'opposition entre le monde de la Direction technique nationale et le monde des entraîneurs exerçant sur le marché des clubs professionnels, dont les représentants étaient Laurent Blanc et Didier Deschamps, membres de l'équipe de France 1998, voire de Jean Tigana, représentant de l'ère Platini. D'ailleurs, dans l'affaire voilà un autre personnage important, grand joueur et actuel président de l'UEFA, la fédération européenne de football, dont on dit qu'il a joué un rôle non négligeable dans la désignation de la France comme organisatrice de l'Euro 2016, mais aussi dans le choix de garder Raymond Domenech après les mauvais résultats de l’Euro 2008.

On peut dire qu'il y a un dysfonctionnement parce que les arguments et les modes de choix de Raymond Domenech sont visiblement dictés par d'autres considérations que la compétence technique, c'est-à-dire qu'ont joué des effets d'appartenance à des réseaux ou qu'on a cherché à défendre des positions acquises et qu'on ne pouvait pas énoncer les raisons du choix : est-ce l'entraîneur qui est en cause ou est-ce la valeur des joueurs ? Peut-être les joueurs étaient-ils moins talentueux qu'on ne voulait bien le dire ? Sûrement étaient-ils des individus différents des générations précédentes et qu'il fallait en tirer des conséquences. Si celles-ci n’ont pas été tirées, c'est peut-être parce que la reconnaissance de l'un ou l'autre de ces aspects mettait en danger la valeur de marché du produit «Équipe de France » et la candidature à l’Euro 2016.

L'intervention politique a participé de cette loi du silence dans la mesure où elle a joué la partition du soutien indéfectible à l'équipe de France comme manifestation d'adhésion à l'unité nationale et en mettant à distance, au nom de l'unité, les critiques faites à Raymond Domenech en les comparant à celles que subissent les ministres. Car le supporter, comme le citoyen, influencé par des commentateurs mal intentionnés, ne peut d'emblée comprendre une action engagée dont les résultats ne seront visibles qu'au bout du chemin où il nous emmène.

Mais enfin, les erreurs et les dysfonctionnements sont inhérents à toute organisation, un fonctionnement parfait n'existe pas ! En effet, les défauts attribués aux joueurs de l'équipe de France, à l'entraîneur ou à la fédération ou les défaillances de communication, de goût du secret et de langue de bois, de manque de patriotisme sont loin d'être le privilège du football. La force des réseaux, la préférence donnée à ceux qui sont les plus proches, la valeur de réputation ou les stratégies égoïstes sont aussi des forces qui, dans le sport autant que dans le monde des médias, de la politique ou des affaires, rendent compte de la reproduction des élites, des choix étroits des milieux professionnels dans leurs recrutements, de la faiblesse de la représentation de la diversité française, qu'elle soit de sexe ou d'origine sociale ou culturelle. Mais le mérite du football, en l'occurrence, est de dramatiser les faits qu'on voit en direct et en temps réel, malgré la bunkérisation de l'équipe de France... ou à cause d'elle ! 


\section{Bunkerisation : oublis et fuites}

Pour qui et pour quoi joue une équipe ? L'impression qu'on tire de cette Coupe du monde est que l'équipe de France a joué pour elle-même, comme si elle n’avait de compte à rendre à personne. Quand on dit " équipe ", il faut entendre les joueurs et l'entraîneur et peut-être même la fédération. " Pour elle-même », c'est-à-dire pour l'équipe, comme s’il n’y avait pas d'enjeux de représentation, comme par exemple de jouer pour des supporters qui ont cette particularité d'être citoyen d'un pays particulier ou de jouer pour le football qui est, comme tous les sports, un jeu où il faut gagner et se donner les moyens sportifs, techniques et tactiques, de le faire et exprimer l'intérêt intrinsèque pris à le faire, et qui est la raison pour laquelle on reçoit des salaires ou des primes élevées ; pour la fédération, jouer pour elle-même, ce serait s’occuper des compromis internes et gérer la marque, comme si le produit "équipe de France » ne dépendait pas de prestations offertes à un public dans une compétition ou de la reconnaissance que les amateurs et les supporters existent, qui ne demandent pas de voter pour élire l'entraîneur, mais de pendre part au jeu en comprenant ce qui se passe.

L’équipe de France s'est coupée de ses supporters, par ses résultats irréguliers et décevants et par des choix problématiques de communication qui traduisent l'indifférence vis-à-vis de ces supporters, le caractère parfaitement accessoire de leur rôle dans la dynamique d'une équipe. Certains enjeux n’ont pas été compris ni intégrés dans les stratégies de communication autour de l'équipe de France, par exemple l'enjeu de la relation de l'équipe et de son entraîneur avec le public. Dans un pays comme la France, où la distance critique vis-àvis du football est au moins égale à l'engagement aveugle, l'art des contre-pieds et du mépris vis-à-vis des supporters jugés incompétents, et de leurs intermédiaires que sont les journalistes sportifs, casse la sorte de contrat qui devrait exister entre un public et l'équipe nationale.

D’autant que nous vivons sous l'ère de la jurisprudence Jacquet qui signifie qu'on ne peut pas critiquer un entraîneur tant qu'on n'a pas vu le résultat de son travail. Elle favorise donc le silence et bien évidemment la recherche des fuites. Cette jurisprudence a donc deux conséquences : elle accroît le pouvoir de communication de l'entraîneur qui pourra développer son style comme il l'entend et mettre en place la stratégie qui lui convient ; elle accroît le pouvoir de nuisance des médias si les relations avec l'équipe de France ne sont pas bonnes. Depuis la Coupe du monde 2006, la stratégie adoptée par Raymond Domenech a été celle du contre-pied permanent, de la contre-vérité et de la bunkerisation de la communication. Cette stratégie oublie qu'un élément déterminant conditionne la stratégie de communication : l'état de concurrence entre les médias dans un contexte de fort intérêt de l'opinion pour les questions de l'équipe de France. On est passé ainsi de l'époque des médias complices, la grande époque d'avant la télévision reine des finances du football, quand n’existent pas les exclusivités, aux médias accusateurs. Comme le disent les anciens, « on savait des choses, mais on savait aussi qu'on ne dirait rien, donc on avait accès à tout ». La loi du silence complice n'est pas nouvelle, mais elle ne peut plus compter sur les médias. Si les uns, la grande télévision hertzienne, sont dépendants des résultats de l'équipe et doivent donc préserver le soutien pour amener le plus de téléspectateurs possibles, il n’en est pas de même des autres télévisions concurrentes et des autres médias qui ont plutôt intérêt à se poser en représentant de l'opinion critique. C'est un effet des concurrences, des crises (notamment de la presse écrite et du principal quotidien sportif, L’Équipe, qui dépend largement du football pour assurer ses ventes), de la gestion de la communication par les sponsors qui travaillent à produire une autre langue de bois et sans doute aussi d'un nouveau type de journalistes sportifs, qui ne sont plus des amoureux du sport, mais des journalistes de scoop et de l'investigation. On pourra, si on travaille à L'Équipe, sur certains 
dossiers, le Tour de France par exemple, être discret sur le dopage, mais être attentif aux turpitudes du sélectionneur ou des joueurs. La stratégie du bunker a donc produit deux effets : elle a incité à rechercher les failles pour traiter des fuites (affaire Zahia, les fameuses insultes des vestiaires de la mi-temps de France-Mexique) et elle a aussi transformé l'équipe de France en émission de télé-réalité.

\section{Que fait l'entraîneur?}

Le résultat des enjeux financiers, des tensions et concurrences à l'intérieur de la fédération est l'autonomisation de l'équipe de France par rapport à d'autres missions de la fédération. De son entraîneur aussi ? Les déclarations des uns et des autres font apparaître qu’il a lui aussi échappé aux procédures ordinaires de contrôle. Ce n'est pas scandaleux en soi car c'est la demande de tous les entraîneurs de pouvoir gérer comme ils l'entendent l'équipe dont ils ont la charge. On peut donc bien comprendre qu'il ait obtenu un pouvoir de décision très fort car il contrôle cette zone d'incertitude fondamentale : il sait comment faire pour gagner, il est celui qui sait ce que les autres ne savent pas, il sait ce qu'il peut faire et ce qu'il ne peut pas faire.

En revanche, il est plus étonnant qu'il ait pu mettre en place sa politique de communication, ou plutôt de contact avec le monde extérieur, concernant l'équipe. C’est le point sur lequel il lui avait été demandé de s’amender à la suite de l'Euro 2008. Mais qui d'autre que l'entraîneur peut parler du jeu de l'équipe ? D’autant qu'il a aussi, comme tous les professionnels, une carrière à négocier et qu'il est peut-être plus pertinent d'imposer une image que d'apparaître comme le bon soldat obéissant. Dans le monde du sport, les acteurs sont soumis à la contrainte de la double performance : ils doivent être bons sportivement et bons médiatiquement pour faire fructifier les gains provenant des succès sportifs. Cela signifie en général qu’il faut se personnaliser, sortir du rôle sportif pour montrer qu'on est aussi doté d'une originalité qui se manifeste dans ses goûts esthétiques, ses investissements sociétaux, son mode de vie. Pour beaucoup, le fait d'entrer dans la société des célébrités et d'en partager les lieux et les objets, vêtements, voitures, sorties, suffit. Ceci principalement pour les jeunes. D’autres feront connaître leur implication dans des causes humanitaires. Ceux qui ont arrêté leur carrière, mais qui restent dans le monde sportif, cumuleront le style de vie maintenu, la culture de sortie, avec la gestion de leur patrimoine s’ils ont investi dans l'immobilier, avec le travail de consultant pour les médias, avec un engagement humanitaire et, pour certains, l'entrée dans la carrière d'entraîneurs.

Pour un entraîneur, la carrière dépend de la réputation accumulée au cours de sa vie de sportif et sa vie d'entraîneur et de la manière de s'appuyer sur un style pour séduire les joueurs et les supporters. Pour Domenech, cela voulait dire réussir avec l'équipe de France pour ensuite entraîner un club professionnel ou entraîner une autre sélection nationale ou peut-être se lancer dans une autre carrière en dehors du football. Ce qui passait par une manière de marquer la distance avec la génération 1998-2000 dont certains membres sont devenus entraîneurs, et les autres consultants ou icônes, tous faisant partie des écuries de quelques agents de joueurs, d'entraîneurs et de célébrités. Être différent des autres ? L'entraîneur est un homme du secret, peu de la parole, mais plutôt du geste et de l'observation, ce qu'étaient les prédécesseurs de Domenech, Jacquet, Lemerre, Santini. Le choix du style personnel, c’est le discours articulé, la belle présentation et la mise à distance ironique qui tranchent avec les manières de s'exprimer en public de ses prédécesseurs. Ce qui a séduit. Mais le style, la volonté de marquer son territoire et la réputation demandent des victoires pour s'imposer. C’est ce qui n’a pas marché et lorsque cela a marché, la finale de la Coupe du monde 2006, on l'a attribué à d'autres, ceux qui ont permis le retour de Zidane et de Makélélé. Confirmé dans ses fonctions après de très fortes critiques et 
connaissant son successeur bien avant que la compétition démarre, on peut penser qu’il a durci son style.

\section{Bunker, loft ou collège de masse ?}

Pourquoi le fiasco ? Laissons ouverte la question et contentons nous de ce qui est visible et qui peut donner prise à quelques éléments de compréhension.

On ne se facilite sans doute pas les choses en faisant vivre des jeunes adultes dans un camp retranché. Faire vivre l'équipe de France, ou n’importe quel groupe, dans une situation d'enfermement favorise le pur jeu des affects et des rapports de force quand la protection contre le monde extérieur, identifié aux seuls médias, ne suffit plus à faire l'unité. On a beaucoup parlé des ego monstrueux des joueurs sans relever le fait que la croyance en sa valeur voire la surestimation de cette valeur touche tous ceux dont l'occupation professionnelle consiste à passer des épreuves de sélection où seul le meilleur l'emporte. C'est le propre de l'économie des stars. Ensuite, il y a sans doute le fait que l'entraîneur perd toute autorité sur le groupe pour différentes raisons : il est déjà mis à l'écart par son employeur ; son absence de succès, son absence de palmarès et son faible prestige comparé aux entraîneurs des clubs où les joueurs évoluent durant l'année ; l'absence de système de jeu et donc l'incertitude sur les rôles attribués à chacun ; un monde extérieur toujours présent grâce aux téléphones mobiles. On a parlé des sportifs trop payés, ce qui n’est pas un privilège des joueurs français et ne peut donc constituer la seule explication de leur comportement. En revanche, l'incertitude sur la bonne utilisation d'un joueur crée des troubles car elle déstabilise l'ensemble valeur sportive/valeur de marché du joueur.

Mais l'équipe de France évoque aussi le collège de masse : tout le monde, surtout les jeunes des classes populaires, y entre, sans qu'on se soucie, par manque d'encadrement et de conscience des enjeux, de contrôler l'acquisition des fondamentaux, techniques et éducatifs. La croyance dans les vertus spontanées du sport fait oublier que l'éducation aux valeurs passe par le travail continu des institutions et de ses membres. Un ancien, mais récent, international a pu dire : «On ne m’a jamais dit dans le centre de formation où j'étais que l'aboutissement idéal de ma vie de footballeur était de jouer une Coupe du monde avec l'équipe de France, on ne m’a jamais dit de bien jouer et de me faire plaisir. »

Enfin, la socialisation des footballeurs a effectivement changé par rapport à la génération de 1998. La massification du football, la rationalisation des modes de sélection des jeunes talents et les espérances de gains d'une carrière dans le football professionnel ont changé radicalement le mode de socialisation des sportifs : non seulement les joueurs ne se recrutent plus en priorité dans les classes populaires traditionnelles, mais dans les nouvelles classes populaires des banlieues, et les joueurs sont soumis à une socialisation où la valeur sportive est indexée immédiatement sur la valeur de marché de l'individu.

La précocité de l'acquisition de la valeur sportive et économique sur le marché international fait entrer les joueurs dans des carrières où, dès l'âge de onze ans, et quelquefois avant, ils se trouvent dans une bulle sportive qui les tient à distance de la réalité ordinaire, notamment des lieux de formation et d'éducation ou du monde du loisir. Passant d'un club à un autre, ils sont les représentants typiques des carrières professionnelles où l'attachement à un collectif apparaît comme un obstacle à la réussite individuelle. Ils sont très tôt l'objet des stratégies des agents ou des familles pour lesquels ils représentent une chance inespérée de promotion sociale. L'existence de clans n'est pas un phénomène nouveau dans le sport, c'est même un fonctionnement de base de ce monde, mais aujourd'hui ces clans dans l'équipe de France renvoient aux affinités existantes ou qui naissent entre ceux qui ont suivi le même parcours vers 
la célébrité sportive et/ou partagent le même style de vie lorsqu'ils se retrouvent dans le loft, ou le bunker, ou "La ferme des célébrités ». Elles sont religieuses (musulman/pentecôtiste), professionnelles (être de Bordeaux ou jouer dans les grands clubs étrangers), de style de vie (être " bringueur » ou non), sociales (venir des quartiers), ethniques (antillais/africains) et, visiblement, elles se croisent quelquefois, sauf si on est enfermé dans le bunker.

Le fiasco de l'équipe de France pose donc des questions redoutables : elles concernent aussi bien les formes de soutien que les individus footballeurs trouvent ou mettent en place pour passer les épreuves qui les mèneront vers le statut de star, que la question des origines et de la place de l'ethnicité dans la société française. Mais au-delà des joueurs, d'autres questions sont posées : la gouvernance des grandes fédérations, la régulation du sport spectacle et la place à attribuer au sport dans la société, la bonne distance que le politique doit avoir vis-à-vis du sport. Comme le disait Jean-Claude Passeron dans un entretien à Esprit : "Attention aux excès de vitesse interprétatifs ${ }^{8}$.»

* Je remercie Georges Vigarello pour les échanges qui ont aidé à écrire cet article.

\section{Notes.}

1. Institut national de l'audiovisuel, « Le baromètre thématique des journaux télévisés », Ina Stat, no 17, juin 2010.

2. Le Monde, mardi 13 juillet 2010.

3. Entretien dans Le Monde, 11-12 juillet 2010.

4.Voir la chronique de Daniel Schneidermann, "Foot : la déroute des automatismes ", Libération, 28 juin 2010.

5.Voir le rapport «Grand Stade » de Philippe Séguin pour préparer la candidature à l’Euro 2016, le rapport d'Éric Besson sur la compétitivité des clubs de football français ou le rapport Costantini sur les grandes salles, produits entre 2007 et 2009.

6. Procès-verbal de l’assemblée générale de la FFF du 19 décembre 2009.

7. Bilan financier de la saison 2008-2009, LFP/DNCG.

8. Jean-Claude Passeron, «Quel regard sur le populaire ? », Esprit, mars-avril 2002. 22 